\title{
New Models of Care and Innovation in Alzheimer's Disease and Related Dementias
}

\author{
An Expert Interview with Anitha Rao, MD, MA
}

Neurocern Inc., Chicago, IL, US

DOl: https://doi.org/10.17925/USN.2018.14.1.19

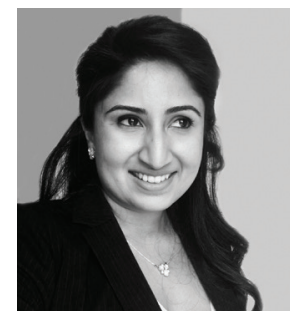

\begin{abstract}
Anitha Rao
Dr. Anitha Rao is a board-certified geriatric neurologist, as well as Chief Executive Officer and founder of Neurocern, a web-based disease management platform that helps enterprise companies manage Alzheimer's disease and related dementias by engaging patients and families with real-time digital assessments and automated care plans. She is one of among 600 dementia experts in the country with subspecialty training in memory disorders, Alzheimer's disease, and dementia. Dr. Rao has published several articles on neurological disease, macroeconomics, and public health outcomes. She has served as an advisor to the American Geriatrics Society, American Academy of Neurology and Women in Bio. She received her medical degree from the University of Toledo, and completed her Neurology residency at Case Western Reserve University, and her fellowship training at the University of California, San Francisco. Dr. Rao also has a Masters in Medical Anthropology from Case Western Reserve University. Her additional interests include health economics, predictive analytics, strategy, and creative writing in medicine.
\end{abstract}

\section{Keywords}

Alzheimer's disease and related dementias (ADRD), digital health, health economics, health informatics, innovation, population health, predictive analytics, public policy, standard of care, technology

Disclosure: Anitha Rao is Chief Executive Officer and founder of Neurocern Inc., and has no other disclosures in relation to this article.

Review Process: This is an expert interview and as such, has not undergone the journal's standard peer review process.

Authorship: The named author meets the International Committee of Medical Journal Editors (ICMJE) criteria for authorship of this manuscript, takes responsibility for the integrity of the work as a whole, and has given final approval for the version to be published.

open Access: This article is published under the Creative Commons Attribution Noncommercial License, which permits any noncommercial use, distribution, adaptation, and reproduction provided the original author and source are given appropriate credit. (c) The Author 2018. Received: February 1, 2018

Published Online: March 14, 2018

Citation: US Neurology. 2018;14(1):19-20

Corresponding Author: Anitha Rao, Neurocern Inc., Chicago, IL, US. E: anitha@neurocern.com

Twitter: @AnithaRF; @neurocern

LinkedIn: linkedin.com/in/anitha-rao-md-neurocern

Support: No funding was received in the publication of this article.
$\mathrm{D}$ r. Anitha Rao delivered several presentations at the Alzheimer's Association International

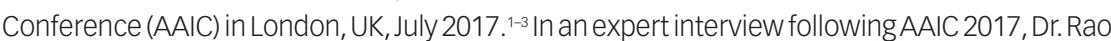
discusses the current limitations of standard care for patients with Alzheimer's disease and related dementias (ADRD), and puts into perspective the potential positive impact of important new technologies such as Neurocern.

\section{Q. What are the major limitations of the current standard of care in patients with Alzheimer's disease and related dementias (ADRD)?}

The shortage of behavioral neurologists and other cognitive specialists has impacted how patients and families access cognitive care for ADRD. According to American Academy of Neurology (AAN) membership data, in the US it is estimated that there are 600 geriatric or behavioral neurologists. Current wait times to see a behavioral neurologist range from 3-6 months in some academic medical centers, representing a critical bottleneck for patients to access timely care. As a result of limited access to specialists, patients and families have been forced to turn to primary care and emergency rooms to manage complex neuro-behavioral symptoms.

Primary care and emergency room providers in many cases are not fully equipped to handle the symptoms, diagnosis, or to provide specialized care instructions to families. The New York Times recently published an article to highlight the need for education reform for ADRD and geriatrics among medical practitioners. ${ }^{4}$ For most medical students, neurology is a 4th-year clerkship rotation that may not be part of their core curriculum. Many students decide medical specialties in their $3 \mathrm{rd}$ year, thereby missing the opportunity to explore a career path in neurology. In primary-care-focused residency programs such as Internal Medicine, neurology is often an optional elective, thereby further limiting exposure to geriatric care.

\section{Q. How does the standard of care for ADRD vary across the US?}

Researchers have demonstrated that specialists including geriatric and behavioral neurologists recognize and diagnose milder stages of dementia earlier than non-specialists. ${ }^{5}$ An earlier diagnosis and access to specialists helps families find answers to their loved one's behavioral changes and reduces unnecessary and costly emergency room visits. In fact, a key study demonstrated patients diagnosed with ADRD by a specialist had lower Medicare costs in the year after diagnosis. ${ }^{5}$

As many physicians recognize, $A D R D$ is a complex clinical condition to manage as it requires a multidisciplinary clinic with social work and nursing support to help families navigate options in care. 
Non-academic hospital systems have been slow to create new dementia service lines due to low reimbursement rates and low clinician supply. As a result, patients in certain regions of the US have very limited options to comprehensive neurological care. This limited access is particularly pronounced in rural and smaller urban centers where multidisciplinary clinics do not presently operate. ${ }^{1}$

\section{Q. Are there implications of ADRD to other clinical specialties?}

Yes, one of the most intriguing areas I have been focusing on is dementia co-existing with other chronic medical conditions. In fact, 95\% of dementia patients have three or more chronic conditions. The costs of care are also much higher when patients have other chronic conditions such as diabetes and heart failure. For example, patients with diabetes and dementia see an $81 \%$ increase in medical costs compared with patients without dementia. ${ }^{5}$ One of the main theories on why this substantial increase occurs is that these chronic conditions require self-management. When cognitive impairment is part of the clinical picture, it can seriously impact how well patients follow disease management instructions and access timely care. It is important that other clinical specialties recognize the implications of cognitive impairment in their assessment and care plans for patients.

\section{Q. How may new technologies like Neurocern be used in ADRD diagnosis and care planning?}

Based on research from the AAN, projections show that the neurology workforce will continue to be significantly undermanned for years to come. New technologies that provide clinical decision support will be extremely valuable for healthcare systems, primary care, and advanced practice providers in the diagnosis and care management of patients with dementia and other neurodegenerative diseases. For federally funded organizations such as the Area Agency of Aging, care planning tools will be key as the number of referrals will continue to grow.

In the international market, neurology supply is even lower than the US, and researchers recently reported that neurological disease is the number one cause of morbidity and mortality worldwide. ${ }^{6}$ Neurologists will be in high demand and new technology like Neurocern offers us unique ways to reach more patients and dramatically improve care.

\section{Q. Could you tell us a little about your journey as a technology entrepreneur?}

Creativity has always been one of my strengths. In medical school, I chose to specialize in neurology because it gave me the freedom to be a creative physician for my patients. Whether it was finding new models of care for stroke, or using technology to help caregivers, neurology provided the perfect blend of imagination and science.
After medical school, I was drawn to pursue a career outside of traditional medicine and knew one day I'd like to work on large public health issues. During my 4th year of medical school, I applied to a Masters program in Medical Anthropology, focusing on cross-cultural aging and dementia. It was during that time I learned how macroeconomic trends influenced access to healthcare. Alzheimer's and dementia topped every public health study I came across in my research as the number one cause of disability worldwide. With the public health statistics in mind, I knew I wanted to specialize in a field that would impact millions of people throughout the world.

After completing my Behavioral Neurology fellowship at the University of California, San Francisco, I was drawn to technology as it suited the creative anthropologist in me. Neurocern Inc. was founded to support family caregivers and improve the outcomes of their loved one's with Alzheimer's and dementia. Neurocern is a web-based disease management platform that helps healthcare companies manage ADRD by engaging patient and families with real-time digital assessments and automated care plans. It has grown over time to support not just families, but the larger ecosystem that cares for dementia patients. It has been truly a journey to understand how hospitals, insurance providers, and other key stakeholders operate in the business world and how impactive technology can not only align their interests but significantly improve patient outcomes.

\section{Q. Technology has greatly impacted neurological care; how can neurologists be involved?}

Certainly, neurology has seen many innovative models of care. Robotics, Gamma Knife ${ }^{\circledast}$ technology, and telestroke have transformed how neurologists reach more patients. It has been truly remarkable to witness this firsthand and neurologists are now on the cutting edge of innovation. Twenty years ago, treating a stroke patient 100 miles away with a robotic telemedicine platform may have seemed fanciful, but advances such as telemedicine have allowed neurologists to increase their impact on patient care.

I would encourage neurologists to get involved in quality improvement projects within their hospitals and learn how to work with members of their respective information technology departments to implement changes in patient care. Outside of the hospital, I'd also encourage neurologists to attend meetups focused on technology, innovation, and entrepreneurship.

This is an exciting time, when advanced technology like Neurocern can drive significant improvements in patient care and I would encourage my colleagues to join me in redefining what it means to treat Alzheimer's disease and related dementias. $\square$

1. Rao A, Manteau-Rao M, Aggarwal NT. Dementia neurology deserts: what are they and where are they located in the US? Alzheimers Dement. 2017;13 Suppl. P509.

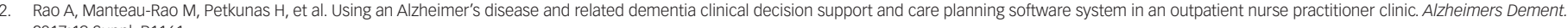
2017;13 Suppl. P1161.

3. Rao A, Manteau-Rao M, Petkunas H, et al. Using neuroscience-based technology to generate clinical decision support and customized care plans for G0505. Alzheimers Dement. 2017:13 Suppl. P1455-6.

4. Cottrell Houle M. An aging population, without the doctors to match. The New York Times. 2015. Available at: www.nytimes.com/2015/09/23/opinion/an-aging-population-without-the-doctors-to-match.html (accessed February 26, 2018).

5. Alzheimer's Association. 2017 Alzheimer's disease facts and figures. Alzheimers Dement. 2017;13:325-73.

6. GBD 2015 Neurological Disorders Collaborator Group. Global, regional, and national burden of neurological disorders during 1990-2015: a systematic analysis for the Global Burden of Disease Study 2015. Lancet Neurol. 2017;16:877-97. 